\title{
Fluctuating interfaces subject to stochastic resetting
}

\author{
Shamik Gupta, Satya N. Majumdar, Grégory Schehr \\ Laboratoire de Physique Théorique et Modèles Statistiques (CNRS UMR 8626), Université Paris-Sud, Orsay, France
}

(Dated: March 1, 2022)

\begin{abstract}
We study one-dimensional fluctuating interfaces of length $L$ where the interface stochastically resets to a fixed initial profile at a constant rate $r$. For finite $r$ in the limit $L \rightarrow \infty$, the system settles into a nonequilibrium stationary state with non-Gaussian interface fluctuations, which we characterize analytically for the Kardar-Parisi-Zhang and Edwards-Wilkinson universality class. Our results are corroborated by numerical simulations. We also discuss the generality of our results for a fluctuating interface in a generic universality class.
\end{abstract}

PACS numbers: 05.40.-a, 05.70.Ln, 02.50.-r

Fluctuating interfaces are paradigmatic nonequilibrium systems commonly encountered in diverse physical situations, e.g., propagation of flame fronts in paper sheets, fluid flow in porous media, vortex lines in disordered superconductors, liquid-crystal turbulence, and many others. Study of such interfaces has many practical applications in the field of molecular beam epitaxy, crystal growth, fluctuating steps on metals, growing bacterial colonies or tumor, etc [1 3 ]. A well-studied model of fluctuating interfaces is the Kardar-Parisi-Zhang (KPZ) equation [4], which is believed to describe a wide class of such out-of-equilibrium growth processes.

Earlier studies of the KPZ equation focused on the universal behavior of the interface roughness, a property which, for instance, in $1+1$ space-time dimensions is characterized by the interface width $W(L, t)$ at time $t$ for an interface growing over a substrate of linear size $L$. It is then known that $W(L, t)$ grows algebraically with time as $t^{\beta}$ for times $t \ll L^{z}$ where $z$ is the dynamic exponent, and saturates for times $t \gg L^{z}$ to a $L$-dependent value $\sim L^{\alpha}$. Here, $\alpha$ is the roughness exponent, while $\beta=\alpha / z$ is the growth exponent. For the KPZ universality class in $1+1$ dimensions, one has $\alpha=1 / 2$ and $z=3 / 2$ [1]3]. More recently, in this case, significant theoretical progress has shown that in the growing regime (i.e., for times $t \ll L^{z}$ ), the notion of universality extends beyond the interface width and holds even for the full interface height distribution at late times $[5-9]$. For example, the scaled cumulative distribution of the interface height fluctuations in a curved (respectively, flat) geometry is described by the so-called Tracy-Widom (TW) distribution $F_{\beta}(x)$, with $\beta=2$ (respectively, $\beta=1$ ). The distribution $F_{2}(x)$ (respectively, $F_{1}(x)$ ) characterizes fluctuations at the edge of the spectrum of random matrices in the Gaussian Unitary Ensemble (GUE)_respectively, Gaussian Orthogonal Ensemble (GOE)] 10, 11]. Height fluctuations measured in experiments on nematic liquid crystals with both curved and flat geometry demonstrated a very good agreement with the TW distributions [12, 13].

One of the first studied models of interface growth is the so-called Eden model [14], which aimed at addressing the growth of bacterial colonies or tumors in mammals.
Such growth typically proceeds through stochastic cell division, and generates an almost compact cell cluster bounded by a rough interface that within the Eden model has scaling properties in the KPZ universality class of interfaces with curved geometry. The growth however may be abruptly interrupted with the cell cluster reduced to its initial size by application of chemicals, as is done, e.g., in chemotherapy to stop the spread of tumor before it becomes life threatening. It is then interesting to study the effects of such random interruptions on the growth process. In this Letter, we show that random interruptions, or random resettings, yield novel steady states with nonGaussian fluctuations which we characterize analytically. Here, we mainly focus on the simpler case of flat interfaces, but our main results can be easily generalized to the case of the curved geometry.

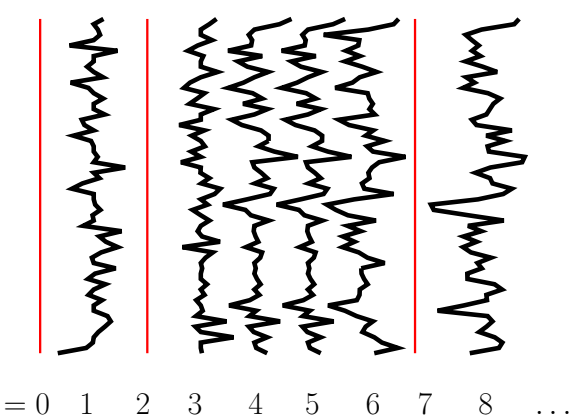

FIG. 1. (Color online) Schematic interface evolution with resetting: Starting from a flat profile, the evolution is interrupted at random times by resetting to the initial configuration from which it recommences.

We consider a $1+1$ dimensional fluctuating interface characterized by a height field $H(x, t)$ at position $x$ and time $t$. Starting from an initially flat profile: $H(x, 0)=0 \forall x$, the heights evolve according to the KPZ equation [4]:

$$
\frac{\partial H}{\partial t}=\nu \frac{\partial^{2} H}{\partial x^{2}}+\frac{\lambda}{2}\left(\frac{\partial H}{\partial x}\right)^{2}+\eta(x, t),
$$

where $\nu$ is the diffusivity, $\lambda$ accounts for the nonlinearities, while $\eta(x, t)$ is a Gaussian noise of zero mean 
and correlations $\left\langle\eta(x, t) \eta\left(x^{\prime}, t^{\prime}\right)\right\rangle=2 D \delta\left(x-x^{\prime}\right) \delta(t-$ $\left.t^{\prime}\right)$. For an interface of length $L$ evolving according to (1), the spatially averaged height $\overline{H(x, t)}=$ $\int_{0}^{L} \mathrm{~d} x H(x, t) / L$ grows with time with velocity $v_{\infty}=$ $(\lambda / 2) \int_{0}^{L} \mathrm{~d} x\left\langle(\partial H / \partial x)^{2}\right\rangle$. The interface width $W \equiv$ $W(L, t)$ is defined as the standard deviation of the height about $\overline{H(x, t)}$. For times larger than a non-universal microscopic timescale $T_{\text {micro }} \sim O(1)$, the width exhibits Family-Vicsek scaling [15]: $W(L, t) \sim L^{\alpha} \mathcal{W}\left(t / T^{*}\right)$, with the crossover timescale $T^{*} \sim L^{z}$ corresponding to the scale over which height fluctuations spreading laterally correlate the entire interface. The scaling function $\mathcal{W}(s)$ behaves as a constant as $s \rightarrow \infty$, and as $s^{\beta}$ as $s \rightarrow 0$. At long times $t \gg T^{*}$, the dynamics of height fluctuations $h(x, t) \equiv H(x, t)-\overline{H(x, t)}$ reaches a nonequilibrium stationary state (NESS) in a finite system, in which the height distribution $P_{\text {st }}(h)$ is a simple Gaussian [1].

Motivated by the situation where the growth is randomly interrupted, e.g., by chemicals, as discussed above, we study the case where the interface is reset at a fixed rate $r$ to the initial flat configuration. The dynamics with random interruptions, shown schematically in Fig. 11. raises a natural question: does it lead to a steady state and if so, can one characterize the distribution of the steady state height fluctuations? Here, we show that indeed random interruptions lead, quite generically, to a nontrivial steady state even in the thermodynamic limit $L \rightarrow \infty$, and for a class of $1+1$ dimensional models including the KPZ interface, we compute analytically the height distribution in this steady state.

Recently, a series of work has shown that resetting dynamics has quite a rich and dramatic effect even on a single particle diffusing in a one-dimensional space $x$ [16 19]. The system involves a random walker undergoing diffusion in presence of resetting, whereby the walker returns to its starting position $x=x_{0}$ at a constant rate $r$. The dynamics models the natural search strategy in which a search for misplaced belongings after continuing in vain for a while recommences by returning to the starting point. While in the absence of resetting, the spatial distribution of the walker is a Gaussian centered at $x_{0}$ with width growing diffusively with time as $\sqrt{t}$, a nonzero $r$ leads to a NESS, with an exponentially decaying profile centered at $x_{0}$ [16 19]. Thus, resetting leads to an otherwise diverging mean search time finite, thereby increasing the efficiency of the search strategy. Random walks with restarts have also been extensively used in computer science as a useful strategy to optimize search algorithms in hard combinatorial problems 20 22].

Our model of resetting of $1+1$ dimensional interface dynamics is a natural extension of the above mentioned single-particle studies to the case of an extended system comprising many interacting degrees of freedom. We note that a recent work also addresses resetting in an extended system, namely, a one-dimensional coagulation-diffusion process, albeit with a different resetting strategy [23].

While in the absence of resetting, the dynamics of interface fluctuations has no steady state in the thermodynamic limit, we demonstrate here that a non-zero resetting rate drives the system to a nontrivial NESS, even in the thermodynamic limit. The NESS obtained is characterized by non-Gaussian interface fluctuations, as we demonstrate analytically. In particular, the stationary interface width $W_{r}$ does not scale with the system size, but instead remains bounded, scaling algebraically with $r, W_{r} \sim r^{-\beta}$, as $r \rightarrow 0$. We discuss our results for fluctuating interfaces belonging to a generic universality class, including the KPZ and the Edwards-Wilkinson (EW) class. Without resetting, the steady state distribution of height fluctuations for both the EW and the KPZ class are identical, and are Gaussian. In contrast, when resetting is switched on, this is not anymore the case, as resetting carries the information of the different growth dynamics of the KPZ and the EW class into the steady state. To support our analysis, we present numerical simulations of the interface dynamics.

We now turn to a derivation of our results. We start with the observation that for times $t \gg T_{\text {micro }}$, when universal scaling behaviors are expected, the dynamics involves two timescales: (i) $T_{\mathrm{r}} \sim 1 / r$, the average time between two consecutive resets, and (ii) the crossover time $T^{*} \sim L^{z}$. Here we consider the case $T_{\mathrm{r}} \ll T^{*}$ (but still $\left.T_{\mathrm{r}} \gg T_{\text {micro }}\right)$, which is easily achieved in the limit of an infinite system, $L \rightarrow \infty$, with finite $r$. In what follows, we set $L \rightarrow \infty$, or equivalently consider time scales $t \ll T^{*}=L^{z}$, such that the asymptotic dynamics is completely governed by the resetting process.

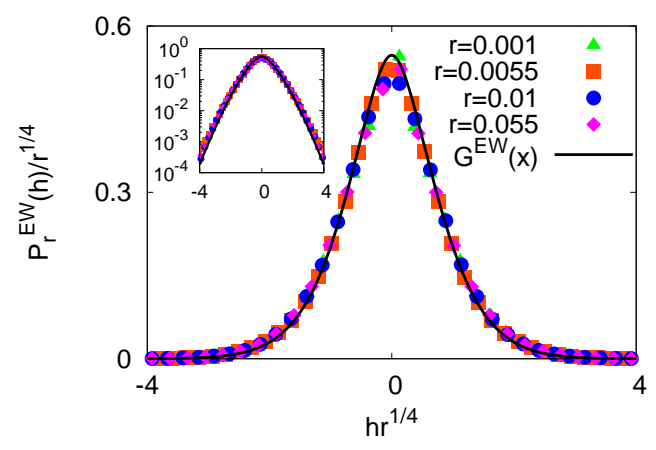

FIG. 2. (Color online) EW interface with resetting: Scaling of the distribution of interface height fluctuations according to Eq. (5), on linear (main plot) and log-linear (inset) scales. With $D=\nu=1$, the points refer to simulation data for $L=2^{14}$, while solid lines for $G^{\mathrm{EW}}(x)$ denote analytical results given by Eq. (6).

In order to compute the height distribution $P^{\text {reset }}(h, t)$ at time $t$ in presence of resetting, we note that the dynamics in the space of configurations is a Markov process. Indeed, let us denote by $\mathcal{C}=\{h(x, t)\}_{0 \leq x \leq L}$ a configura- 
tion of the whole system of size $L$. The KPZ equation (1) implies that in the time interval between two successive resetting events, the dynamics of the "vector" $\mathcal{C}$, whose entries are labelled by the space position $x$, is Markovian. The dynamics in configuration space is thus a renewal process. Then, at some fixed time $t$, let the time elapsed since the last renewal be in the interval $[\tau, \tau+\mathrm{d} \tau]$, with $0 \leq \tau \leq t$. Noting that the probability for this latter event is $r e^{-r \tau} \mathrm{d} \tau$, we have

$$
P^{\text {reset }}(\mathcal{C}, t)=\int_{0}^{t} \mathrm{~d} \tau r e^{-r \tau} P(\mathcal{C}, \tau)+e^{-r t} P(\mathcal{C}, t) .
$$

Here, $P^{\text {reset }}(\mathcal{C}, t)$ (respectively, $P(\mathcal{C}, t)$ ) is the probability to be in configuration $\mathcal{C}$ at time $t$, starting from an initially flat interface in the presence (respectively, absence) of any resetting. The last term on the right hand side (rhs) of (2) accounts for the event when there has not been a single resetting event in the time interval $[0, t]$. Integrating both sides of Eq. (2) over all the possible configurations $\mathcal{C}$, and noting that $P(\mathcal{C}, \tau)$ is normalized to unity for every $\tau$, we check that $P^{\text {reset }}(\mathcal{C}, t)$ for every $t$ is also normalized. The dynamics is Markovian in the configuration space, but is not so for the relative height $h(x, t)$ at a given point $x$ due to the presence of space derivatives of the height field on the rhs of (1) 24]. Nevertheless, Eq. (2) being linear, one obtains the marginal distribution $P^{\text {reset }}(h, t)$ of the height field $h(x, t)$ by integrating this equation over heights $h(y, t)$ at positions $y \neq x$ :

$$
P^{\text {reset }}(h, t)=\int_{0}^{t} \mathrm{~d} \tau r e^{-r \tau} P(h, \tau)+e^{-r t} P(h, t),
$$

where $P(h, t)$ is the height distribution in the absence of resetting, starting from a flat initial configuration. In the limit $t \rightarrow \infty$, we see from (3) that the system reaches a steady state characterized by the distribution

$$
P_{\mathrm{r}}(h)=P^{\text {reset }}(h, t \rightarrow \infty)=\int_{0}^{\infty} \mathrm{d} \tau r e^{-r \tau} P(h, \tau),
$$

an exact result valid for any $r$ and $h$. Note that due to resetting, a non-local probability flux exists only from all $h \neq 0$ values to $h=0$. This leads to a circulation of probability between a source at $h=0$ and several sinks at $h \neq 0$, so that the steady state reached is a NESS.

We consider first the simpler EW equation which corresponds to $\lambda=0$ in Eq. (1), thereby leading to an evolution linear in $h$ [25]. In this case, $v_{\infty}=0$ and the Family-Vicsek scaling holds with the EW exponents $\alpha=1 / 2$ and $z=2$. Without resetting, the steady state distribution $P_{\mathrm{st}}(h)$ at times $t \gg T^{*}$ in a finite system is Gaussian, and is in equilibrium, in contrast to the NESS of a KPZ interface. For times $t \ll T^{*}$, the interface distribution is also Gaussian, but with a non-stationary width $W^{\mathrm{EW}}(t)=D \sqrt{2 t /(\pi \nu)}$, for all $t$. Hence, plugging this Gaussian form for $P(h, \tau)$ into Eq. (4), we find that $P_{\mathrm{r}}^{\mathrm{EW}}(h)$ has the scaling form

$$
P_{\mathrm{r}}^{\mathrm{EW}}(h) \sim \sqrt{\gamma} r^{1 / 4} G^{\mathrm{EW}}\left(h \sqrt{\gamma} r^{1 / 4}\right),
$$

where $\gamma=\sqrt{\pi \nu} /\left(D 2^{3 / 2}\right)$ and $G^{\mathrm{EW}}(x)$ is given by

$$
G^{\mathrm{EW}}(x)=\frac{1}{\sqrt{\pi}} \int_{0}^{\infty} \frac{\mathrm{d} y}{y^{1 / 4}} \exp \left(-y-\frac{x^{2}}{\sqrt{y}}\right),
$$

which is symmetric in $x, G^{\mathrm{EW}}(-x)=G^{\mathrm{EW}}(x)$. From the scaling form in (5), one obtains the scaling of the stationary width with $r$ as $W_{\mathrm{r}}^{\mathrm{EW}} \sim r^{-1 / 4}[26]$. The integral in (6) can be expressed in terms of hypergeometric series. In particular, $G^{\mathrm{EW}}(x)$ behaves asymptotically as

$G^{\mathrm{EW}}(x) \sim\left\{\begin{array}{l}\frac{1}{\sqrt{\pi}}\left[\Gamma\left(\frac{3}{4}\right)-x^{2} \Gamma\left(\frac{1}{4}\right)+\frac{8}{3} \sqrt{\pi}|x|^{3}\right], x \rightarrow 0, \\ c|x| \exp \left[-3 / 2^{2 / 3}|x|^{4 / 3}\right], x \rightarrow \pm \infty,\end{array}\right.$

where $\Gamma(x)$ is the Gamma function and $c$ is a computable constant. Interestingly, due to the $|x|^{3}$ term in (77), $G^{\mathrm{EW}}(x)$ is non-analytic close to $x=0$. In the limit $x \rightarrow \pm \infty$, the stretched exponential behavior (77) is significantly different from a Gaussian tail.

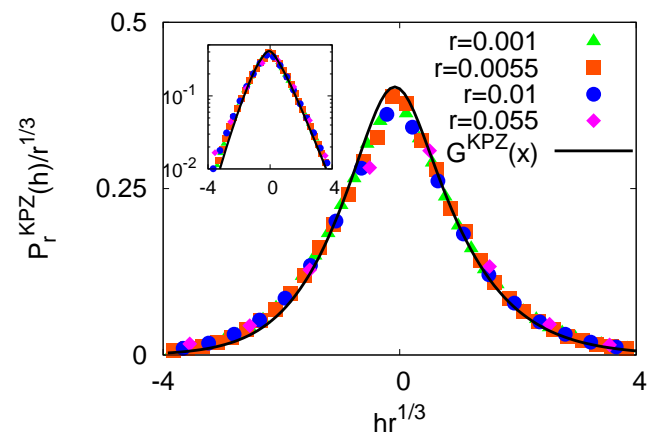

FIG. 3. (Color online) KPZ interface with resetting: Scaling of the distribution of interface height fluctuations according to Eq. (11), on linear (main plot) and log-linear (inset) scales. Here, points refer to simulation data for $L=2^{15}$, while solid lines for $G^{\mathrm{KPZ}}(x)$ denote analytical results given by Eq. (12).

In order to check our prediction (5), we now report on results of numerical simulations performed for a discrete one-dimensional periodic interface $\left\{H_{i}(t)\right\}_{i=1,2, \ldots, L}$ evolving in times $t_{n}=n \Delta t$, with $n$ an integer and $\Delta t \ll 1$. Starting from a flat interface, $H_{i}(0)=0 \forall i$, the interface at time step $t_{n}$ is reset to its initial configuration with probability $r \Delta t$, and updated according to the EW dynamics with probability $1-r \Delta t$. The results shown in Fig. 2 illustrate a very good agreement with. Evidently, $P_{\mathrm{r}}^{\mathrm{EW}}(h)$ is highly non-Gaussian.

We now turn to the KPZ case. Here, it is known that for times $T_{\text {micro }} \ll t \ll T^{*}$, and for a flat initial profile, the interface height $H(x, t)$ has a deterministic linear growth with stochastic $t^{1 / 3}$ fluctuations [9]:

$$
H(x, t)=v_{\infty} t+(\Gamma t)^{1 / 3} \chi(x) .
$$


Here, $\Gamma \equiv \Gamma(\nu, \lambda, D)$ is a constant, while $\chi$ is a timeindependent random variable distributed according to the TW distribution corresponding to GOE, $f_{1}(\chi)=$ $F_{1}^{\prime}(\chi)$, which can be written explicitly in terms of the Hastings-McLeod solution of the Painlevé II equation [10]. In particular, $f_{1}(\chi)$ has asymmetric nonGaussian tails [10, 27]: $f_{1}(\chi) \approx \exp \left(-|\chi|^{3} / 24\right)$ as $\chi \rightarrow$ $-\infty$, while $f_{1}(\chi) \approx \exp \left(-2 \chi^{3 / 2} / 3\right)$ as $\chi \rightarrow \infty$ [28].

From Eq. (8), we get

$$
h=(\Gamma t)^{1 / 3}\left[\chi-\frac{1}{L} \int_{0}^{L} \mathrm{~d} x \chi(x)\right] .
$$

Knowing that $f_{1}(\chi)$ has a finite mean $\langle\chi\rangle<0$, it follows from the law of large numbers that in the limit $L \rightarrow \infty$, the second term on the rhs converges to $\langle\chi\rangle$, so that $\langle h\rangle=0$. In this case, in the limit $\tau \rightarrow \infty, h \rightarrow \infty$, keeping $h / \tau^{1 / 3}$ fixed, $P(h, \tau)$ takes the scaling form

$$
P(h, \tau) \sim \frac{1}{(\Gamma \tau)^{1 / 3}} \tilde{f}_{1}\left(\frac{h}{(\Gamma \tau)^{1 / 3}}\right),
$$

where $\widetilde{f}_{1}(x) \equiv f_{1}(x+\langle\chi\rangle)$. From Eq. (4), we see that in the limit $r \rightarrow 0, P_{\mathrm{r}}^{\mathrm{KPZ}}(h)$ has a universal scaling form, as follows from the fact that the integral (4) in this limit is dominated by the limit $\tau \rightarrow \infty$ where $P(h, \tau)$ can be replaced by its scaling form (10) for $h \rightarrow \infty$, keeping $h / \tau^{1 / 3}$ fixed. Hence, for $r \rightarrow 0, h \rightarrow \infty$, keeping $h r^{1 / 3}$ fixed, we get

$$
P_{\mathrm{r}}^{\mathrm{KPZ}}(h) \sim\left(r \Gamma^{-1}\right)^{1 / 3} G^{\mathrm{KPZ}}\left[\left(r \Gamma^{-1}\right)^{1 / 3} h\right],
$$

where the scaling function $G^{\mathrm{KPZ}}(x)$ is given by

$$
G^{\mathrm{KPZ}}(x)=\int_{0}^{\infty} \mathrm{d} y \frac{e^{-y}}{y^{1 / 3}} \widetilde{f}_{1}\left(\frac{x}{y^{1 / 3}}\right) .
$$

At variance with $G^{\mathrm{EW}}(x)$, the function $G^{\mathrm{KPZ}}(x)$ is not symmetric in $x$. On the other hand, since $\tilde{f}_{1}$ has zero mean, and has fatter tails as $x \rightarrow \infty$ than as $x \rightarrow-\infty$, $G^{\mathrm{KPZ}}(x)$ has its maximum at a negative value $x^{*}<0$. From (11), the stationary width scales with $r$ as $W_{\mathrm{r}}^{\mathrm{KPZ}} \sim$ $r^{-1 / 3}$. One can check in this case that $G^{\mathrm{KPZ}}(x)$ is analytic close to $x^{*}$. Its asymptotic behaviors for $x \rightarrow \pm \infty$, obtained from the corresponding behaviors of $\tilde{f}_{1}(x)$ combined with a saddle point analysis, are

$$
G^{\mathrm{KPZ}}(x) \approx\left\{\begin{array}{l}
\exp \left(-|x|^{3 / 2} / \sqrt{6}\right), x \rightarrow-\infty \\
\exp \left(-3^{1 / 3} x\right), x \rightarrow+\infty
\end{array}\right.
$$

From (12), we see that $G^{\mathrm{KPZ}}(x)$ has a non-analytic behavior as $x \rightarrow 0$, where $G^{\mathrm{KPZ}}(x) \sim A+B x+C x^{2} \ln x$, with $A, B, C$ being constants. This non-analyticity of the steady-state distribution at the value to which the system is reset (here, $x=h=0$, see Fig. 1) also occurs for EW interfaces (7), as well as in the case of random walks with resetting [16, 17]. A comparison between Eqs. (77) and
(13) shows that, for $r>0$, the steady state height fluctuations for EW and KPZ dynamics are quite different. This is in contrast to the case without resetting where they are identical and Gaussian.

To confirm the scaling form (11), we performed numerical simulations of a discrete one-dimensional periodic interface $\left\{H_{i}(t)\right\}_{1 \leq i \leq L}$ evolving in discrete times $t$. The interface is reset to the initial flat configuration with probability $r$, and updated with probability $1-r$ according to the following dynamics of the ballistic deposition model which is in the KPZ universality class [1-3]:

$$
H_{i}(t+1)=\max \left[H_{i-1}(t), H_{i}(t)+1, H_{i+1}(t)\right] .
$$

Comparison between simulations and theory in Fig. 3 shows a very good agreement. Note that for the comparison, one has to compute the integral in (12) by using the TW GOE distribution whose mean has been shifted to zero, and then scale the data by a model-dependent fitting parameter that plays the role of $\Gamma$ in (8). We see that as for the EW case, $P_{\mathrm{r}}(h)$ is non-Gaussian.

For the case of a general interface characterized by scaling exponents $\alpha, z$, and $\beta=\alpha / z$, we now give scaling arguments to compute $P_{\mathrm{r}}(h)$. In the limit $\tau \rightarrow \infty$ and $h \rightarrow \infty$, keeping $h / \tau^{\beta}$ fixed, the height distribution $P(h, \tau)$ quite generally has the scaling form

$$
P(h, \tau) \sim \tau^{-\beta} g\left(h \tau^{-\beta}\right) .
$$

In this case, the distribution $P_{\mathrm{r}}(h)$ is universal in the limit $r \rightarrow 0$ and $h \rightarrow \infty$, keeping $h r^{\beta}$ fixed [see the discussion following Eq. (10)]. The associated scaling function is obtained by plugging the behavior in (15) into Eq. (4), which yields

$$
P_{\mathrm{r}}(h) \sim r^{\beta} G\left(h r^{\beta}\right), G(x)=\int_{0}^{\infty} \frac{d y}{y^{\beta}} e^{-y} g\left(\frac{x}{y^{\beta}}\right),
$$

implying in particular the behavior of the stationary width $W_{\mathrm{r}} \sim r^{-\beta}$ as $r \rightarrow 0$. The $\mathrm{EW}$ and KPZ interfaces correspond to $\beta=1 / 4$ and $\beta=1 / 3$ respectively. In the generic case when $g(x) \sim \exp \left(-a x^{\gamma_{ \pm}}\right)$ as $x \rightarrow \pm \infty$, one obtains by a saddle point analysis of (16) that $G(x) \sim \exp \left(-b x^{\nu_{ \pm}}\right)$as $x \rightarrow \pm \infty$ with $\nu_{ \pm}=\gamma_{ \pm} /\left(1+\beta \gamma_{ \pm}\right)$. Note also that (16) implies that $G(x)$ is generically non-analytic at $x=0$.

To conclude, we studied in this work one-dimensional fluctuating interfaces of length $L$ with the interface stochastically resetting to a fixed initial profile at a constant rate $r$. For finite $r$ in the limit $L \rightarrow \infty$, the system reaches at long times a nonequilibrium stationary state with non-Gaussian interface fluctuations. We characterized these fluctuations analytically for the KPZ and EW universality class and verified these predictions via numerical simulations. For simplicity, we focused here on interfaces in a flat geometry, though our results can be easily extended to the case of a curved geometry. Also, 
our results could possibly be verified in experiments, in particular in the recent ones on liquid crystals [12, 13].

Acknowledgements: We acknowledge support by the ANR grant 2011-BS04-013-01 WALKMAT and in part by the Indo-French Centre for the Promotion of Advanced Research under Project 4604-3.

[1] A.-L. Barabási and H. E. Stanley, Fractal concepts in surface growth (Cambridge University Press, 1995).

[2] T. Halpin-Healy and Y.C. Zhang, Phys. Rep. 254, 215 (1995).

[3] J. Krug, Adv. Phys. 46, 139 (1997).

[4] M. Kardar, G. Parisi, and Y.-C. Zhang, Phys. Rev. Lett. 56, 889 (1986).

[5] J. Baik, P. Deift, and K. Johansson, J. Am. Math. Soc. 12, 1119 (1999).

[6] M. Prähofer and H. Spohn, Phys. Rev. Lett. 84, 4882 (2000).

[7] K. Johansson, Comm. Math. Phys. 209, 437 (2000).

[8] S. N. Majumdar, in Complex Systems, Volume LXXXV: Lecture Notes of the Les Houches Summer School, edited by J.-P. Bouchaud, M. Mézard, and J. Dalibard (Elsevier, 2007) [arXiv:cond-mat/0701193].

[9] T. Sasamoto and H. Spohn, Phys. Rev. Lett. 104, 230602 (2010); T. Sasamoto and H. Spohn, Nucl. Phys. B 834, 523 (2010); P. Calabrese, P. Le Doussal, and A. Rosso, Europhys. Lett. 90, 20002 (2010); V. Dotsenko, Europhys. Lett. 90, 20003 (2010); G. Amir, I. Corwin, and J. Quastel, Comm. Pure and Appl. Math. 64, 466 (2011).

[10] C. A. Tracy and H. Widom, Comm. Math. Phys. 177, 727 (1996).
[11] C. A. Tracy and H. Widom, Comm. Math. Phys. 159, 151 (1994).

[12] K. A. Takeuchi and M. Sano, Phys. Rev. Lett. 104, 230601 (2010).

[13] K. A. Takeuchi, M. Sano, T. Sasamoto, and H. Spohn, Sci. Rep. (Nature) 1, 34 (2011).

[14] M. Eden, Proc. 4th Berkeley Symp. Math. Stat. Prob. 4, 233 (1961).

[15] F. Family and V. Vicsek, J. Phys. A 18, L75 (1985).

[16] M. R. Evans and S. N. Majumdar, Phys. Rev. Lett. 106, 160601 (2011).

[17] M. R. Evans and S. N. Majumdar, J. Phys. A: Math. Theor. 44, 435001 (2011).

[18] M. R. Evans, S. N. Majumdar, and K. Mallick, J. Phys. A: Math. Theor. 46, 185001 (2013).

[19] J. Whitehouse, M. R. Evans, and S. N. Majumdar, Phys. Rev. E 87, 022118 (2013).

[20] L. Lovasz, Random walks on graphs: A survey, in Combinatronics (Bolyai Society for Mathematical Studies, 1996), Vol. 2, p. 1.

[21] A. Montanari and R. Zecchina, Phys. Rev. Lett. 88, 178701 (2002).

[22] I. Konstas, V. Stathopoulos, and J. M. Jose, in Proc. of the 32nd International ACM SIGIR Conference (ACM, New York, 2009), p. 195.

[23] X. Durang, M. Henkel, and H. Park, arXiv:1309.2107.

[24] A. J. Bray, S. N. Majumdar, and G. Schehr, Adv. Phys. 62, 225 (2013).

[25] S. F. Edwards and D. R. Wilkinson, Proc. R. Soc. London Ser. A 381, 17 (1982).

[26] This result is valid here for all $r$, not necessarily small, since $P(h, \tau)$ is a Gaussian.

[27] J. Baik, R. Buckingham, and J. DiFranco, Comm. Math. Phys. 280, 463 (2008).

[28] We use the symbol $\approx$ to mean logarithmic equivalence. 